\title{
Optimizing the management of HER2-negative metastatic breast cancer in the era of PARP inhibitors - proceedings from breast cancer expert group meeting
}

\author{
Shaheenah Dawood ${ }^{1}$, Maria Konstantinova ${ }^{2}$, Florencia Perazzo ${ }^{3}$, Sung-Bae Kim ${ }^{4}$, \\ Cynthia Villarreal-Garza ${ }^{5,6}$, Sandra Ximena Franco ${ }^{7}$, Sergio D. Simon ${ }^{8}$, Tamer El-Nahas ${ }^{9}$ \\ ${ }^{1}$ Department of Medical Oncology, Mediclinic City Hospital - North Wing, Mohammed Bin Rashid University of Medicine and Health Sciences, \\ Dubai Health Care City, Dubai, United Arab Emirates; ${ }^{2}$ Department of Antitumor Drug Therapy, F. VladimirskIy Moscow Regional Research \\ Clinical Institute (MONIKI), Moscow, Russia; ${ }^{3}$ Department of Oncology, Centro de Educación Médicae Investigaciones Clínicas (CEMIC), \\ Ciudad De Buenos Aires, Argentina; ${ }^{4}$ Department of Oncology, Asan Medical Center, University of Ulsan College of Medicine, Songpa-gu, Seoul, \\ Korea; ${ }^{5}$ Centro de Cancer de Mama, Hospital Zambrano Hellion, Tecnologico de Monterrey, Mexico; ${ }^{6}$ Depto. de Investigacion, Instituto Nacional \\ de Cancerologia, Tlalpan, Ciudad de México; ${ }^{7}$ Department of Oncology, Clínica del Country, Bogotá, Colombia, Colombia; ${ }^{8}$ Centro Paulista de \\ Oncologia (CPO), Faria Lima, 4300 - Vila Olímpia, São Paulo, Brazil; ${ }^{9}$ Kasr Al Ainy Hospital, Cairo university, Giza, Egypt \\ Contributions: (I) Conception and design: All authors; (II) Administrative support: None; (III) Provision of study materials or patients: All authors; \\ (IV) Collection and assembly of data: All authors; (V) Data analysis and interpretation: All authors; (VI) Manuscript writing: All authors; (VII) Final \\ approval of manuscript: All authors. \\ Correspondence to: Shaheenah Dawood. Consultant Medical Oncologist, Assoc. Professor of Clinical Oncology, Mediclinic City Hospital - \\ North Wing, Mohammed Bin Rashid University of Medicine and Health Sciences, Dubai Health Care City, Dubai, United Arab Emirates. \\ Email: shaheenah@post.harvard.edu.
}

\begin{abstract}
The therapeutic landscape of human epidermal growth factor receptor 2-negative (HER2-) metastatic breast cancer $(\mathrm{mBC})$ has evolved considerably with the introduction of newer targeted agents such as poly-ADP ribose polymerase inhibitors (PARPi), novel chemotherapeutic agents, immunotherapy, and endocrine therapies. In this scenario, optimizing the appropriate treatment sequence is a daunting task for clinicians. To develop evidence-based answers to key clinical questions on treatment selection and appropriate treatment sequence for the management of patients with HER2- mBC in the era of PARPi, a breast cancer expert group meeting was convened. The expert panel comprised of eight key opinion leaders from Argentina, Brazil, Colombia, Egypt, Mexico, Moscow, South Korea, and the United Arab Emirates, who convened and reviewed the literature, discussed the clinical practices across the participating regions, and formulated answers to key clinical questions for optimizing the management of HER2- mBC. In this review, evidence-based answers have been provided pertaining to (I) the specific $\mathrm{mBC}$ population to be considered for BRCA testing, optimal time point of $B R C A$ testing, and genetic counselling in $\mathrm{mBC}$ patients; (II) the role of PARPi versus platinum therapy in HER2 - mBC patients in the metastatic setting; (III) sequencing treatment in metastatic triple-negative breast cancer (TNBC) and hormone receptor-positive HER2- mBC patients, and defining the place of PARPi in the sequencing algorithms; and (IV) the need for a breast cancer registry for patients with HER2- $\mathrm{mBC}$. This expert review will serve as a comprehensive guide to clinicians for optimizing BRCA testing and managing patients with BRCA mutation $(B R C A \mathrm{~m})$ and HER2- mBC. The data collected from the proposed HER2 - mBC registry will help understand the treatment practices, identify unmet needs, and develop strategic policies regionally to help improve access to optimized care of HER2- mBC.
\end{abstract}

Keywords: Human epidermal growth factor receptor; breast cancer; $B R C A$ mutations; poly-ADP ribose polymerase inhibitors (PARPi); immunotherapy

Submitted Apr 10, 2020. Accepted for publication Jul 28, 2020.

doi: $10.21037 / \mathrm{cco}-20-138$

View this article at: http://dx.doi.org/10.21037/cco-20-138

(c) Chinese Clinical Oncology. All rights reserved.

Chin Clin Oncol 2020;9(5):61 I http://dx.doi.org/10.21037/cco-20-138 


\section{Introduction}

Breast cancer was the most commonly diagnosed cancer $(24.2 \%)$ and the leading cause of cancer death among women worldwide (15\%) in 2018 (1). The high mortality rate of breast cancer has been associated with complications resulting from advanced disease. While about $6 \%$ of patients will present with metastatic disease at the time of diagnosis (2), approximately $30 \%$ of women initially diagnosed with early-stage breast cancer ultimately progress to metastatic breast cancer ( $\mathrm{mBC}$ ) (3). The prognosis of $\mathrm{mBC}$ is poor compared to localized (early) breast cancer; the 5-year survival rate obtained from the Surveillance, Epidemiology, and End Results Program (SEER) 2018 data for early and metastatic breast cancer was $98.8 \%$ and $27.4 \%$, respectively (2).

Breast cancer may be conventionally classified into triplenegative breast cancer (TNBC) [hormone receptor-negative (HR-), human epidermal growth factor receptor-negative (HER2-) breast cancer (accounting for $15 \%$ of cases)], HRpositive [HR+/HER2 - or HR+/HER2+ (70\%)], and HER2positive [HR-/HER2+ (15\%)] breast cancer (4). Among the breast cancer subtypes, HR+/HER2- is the most common, followed by TNBC with an age-adjusted rate of 85.8 and 13 new cases per 100,000 women, respectively (5).

In view of the high incidence and mortality associated with HER2 - mBC, the focus of research has been to elucidate the factors influencing the prognosis of these tumors and targeted treatments that would improve the prognostic outcomes in these patients. Genetic mutations in BRCA1 and BRCA2 genes have a role in predicting the risk of development of disease and have been shown to serve as a biomarker of response to targeted agents. Thus, the treatment landscape of HER2 - mBC has changed dramatically with the introduction of several new promising targeted regimens such as poly-ADP ribose polymerase inhibitors (PARPi) with significant anti-tumor efficacy when used in individuals with $B R C A 1 / 2$ or homologous recombination deficiency (HRD) mutations in the metastatic setting (6). Therefore, optimization of treatment selection and sequencing will be crucial to help guide clinical decisions, given the plethora of emerging options available in the therapeutic armamentarium of HER2 - mBC.

\section{Rationale and objectives of the expert group meeting}

An expert panel of eight key opinion leaders from
Argentina, Brazil, Colombia, Egypt, Mexico, Moscow, South Korea, and the United Arab Emirates convened in September 2019 in Barcelona, Spain to: (I) obtain expert opinion on factors affecting treatment selection and sequencing for the management of HER2- mBC; (II) discuss and formulate answers to key clinical questions for optimizing the treatment of HER2 - mBC; (III) propose treatment sequencing algorithms in TNBC and $\mathrm{HR}+$ / HER2 - mBC (endocrine sensitive and endocrine resistant) settings, along with defining the place of PARPi in these algorithms; (IV) understand the current landscape for $B R C A$ testing in breast cancer, including challenges, guidelines and ways to optimize genetic testing; and (V) review the feasibility of the development of a regional breast cancer registry.

\section{Overview of clinical evidence for the management of HER2- mBC with PARP inhibitors}

\section{Overview of PARP inbibitors}

Poly-ADP ribose polymerase is a family of enzymes that play a key role in DNA transcription and repair of DNAdamage by base excision repair (BER) process. Both catalytic inhibition of PARP and PARP trapping are promising strategies that selectively target cancer cells with germline mutations in BRCA1/2 genes involved in homologous recombination repair (HRR) of DNA-damage. This may contribute to the efficacy of PARPi in the treatment of patients with breast cancer (7).

\section{Phase II clinical evidence}

A prospective non-randomized, phase II clinical trial examined the efficacy and safety of the PARPi, olaparib as monotherapy (400 $\mathrm{mg}$ twice daily) in patients with advanced ovarian, breast, pancreatic and prostate cancers, associated with germline BRCA1/2 (gBRCA1/2) mutations. Out of 62 heavily pre-treated $\mathrm{mBC}$ patients $(\geq 3$ prior chemotherapy regimens), the primary endpoint of tumor response rate [or objective response rate (ORR)] was observed in 8 $(12.9 \%)$ patients. Patients with $\mathrm{mBC}$ with prior platinum exposure had a response rate of $9.5 \%$ as compared with $20 \%$ in patients without prior platinum exposure. Median progression-free survival (PFS) was 3.7 months, and median overall survival (OS) was 11 months (8). Another phase II study assessing the efficacy, safety and tolerability of olaparib (400 mg twice daily) in 27 patients 
with $B R C A 1 / 2$ mutations and $\mathrm{mBC}(\geq 3$ lines of prior therapy failure) provided a positive proof of concept for PARP inhibition in these patients. The primary endpoint, ORR, was $41 \%$. There was an increased shrinkage in tumor size from baseline $(-30 \%)$, depicting a favourable response with the olaparib-targeted treatment strategy in patients with $B R C A$-associated $\mathrm{mBC}$ (6). The activity of another PARPi, talazoparib was assessed in platinum-treated (cohort 1) or cytotoxic non-platinum (cohort 2)-pre-treated $\mathrm{mBC}$ patients with $\mathrm{g} B R C A 1 / 2$ mutations $(\mathrm{n}=84)$ in the phase II ABRAZO study. Confirmed ORR was $21 \%$ and $37 \%$ in cohorts 1 and 2, respectively; the corresponding median duration of response was 5.8 and 3.8 months. Exploratory analysis revealed a higher response rate in patients with longer platinum-free interval in cohort 1 (9).

\section{Phase III clinical evidence}

\section{OLYMPIAD trial}

\section{Study design}

The OlympiAD trial was designed to compare the efficacy and safety of olaparib with that of the standard therapy with single-agent chemotherapy of physician's choice (capecitabine, eribulin, or vinorelbine) among patients with HER2 - (HR-/HER2 - or HR+/HER2-) mBC and a confirmed or suspected deleterious $\mathrm{g} B R C A$ mutation. A total of 302 eligible patients were randomized in a 2:1 ratio: 205 patients received olaparib tablets $(300 \mathrm{mg}$ twicedaily) and 91 patients received standard physician's choice chemotherapy in 21-day cycles. The primary endpoint was PFS based on blinded independent central review and analyzed on an intention-to-treat (ITT) basis. Additional data on OS, ORR, complete response (CR), partial response $(\mathrm{PR})$ and median duration of response were collected for prespecified secondary endpoints (10).

\section{Study results}

The median age of patients was 44 years. After a median follow-up of 14 months, median PFS in HER2- mBC patients was 2.8 months longer in the olaparib group compared to the standard therapy group [7.0 vs. 4.2 months; hazard ratio (HR): $0.58 ; \mathrm{P}<0.001]$. The PFS benefit was consistent, regardless of prior platinum therapy or baseline BRCA1/2 mutations but was more evident among TNBC versus HR+/HER2 - mBC patients (10). Furthermore, in comparison with the standard therapy, olaparib showed PFS benefits in patients with liver metastases and central nervous system (CNS) metastases (11).

There was an improvement in the secondary endpoints: the ORR in the olaparib group was approximately double the rate in the standard therapy group (59.9\% versus $28.8 \%$ ), and CR and PR were achieved in a higher proportion of patients in the olaparib group as compared to the standard therapy group $(9 \% v s .1 .5 \%$ and $51 \% v s$. $27.3 \%$, respectively). In patients with HR+/HER2- mBC, the ORR was $65.4 \%$ in the olaparib group and $36.4 \%$ in the standard therapy group. Among TNBC patients, the ORR was $54.7 \%$ and $21.2 \%$ in the olaparib and standard therapy groups, respectively. The median duration of response was 6.4 months in the olaparib group and 7.1 months in the standard therapy group (10).

The OLYMPIAD study was not powered to detect differences in treatment effect between subgroups, and no significant difference in OS was observed between the olaparib versus the standard therapy group (19.3 vs. 17.1 months; $\mathrm{P}=0.513$ ) (12). However, a significant benefit in OS was observed in patients with no prior chemotherapy for $\mathrm{mBC}$ (22.6 months with olaparib vs. 14.7 months in the standard therapy group; $\mathrm{P}=0.02)(12)$. Based on the results of the OLYMPIAD trial, olaparib became the first PARPi to be approved by the Food and Drug Administration (FDA) for $\mathrm{mBC}$ with $\mathrm{g} B R C A$ mutations.

\section{EMBRACA trial}

\section{Study design}

This trial was similar to the OLYMPIAD trial in terms of design. A total of 431 eligible patients with $\mathrm{mBC}$ and $\mathrm{g} B R C A$ mutations were randomized in a 2:1 ratio and stratified by receptor status, the extent of prior therapy and CNS metastases, to receive the PARPi, talazoparib (1 mg PO daily) or standard single chemotherapy agent of physician's choice (capecitabine, eribulin, gemcitabine or vinorelbine) in 21-day cycles. The primary objective was PFS assessed by blinded independent central review. Secondary objectives were OS, ORR, CR and median duration of response (13).

\section{Study results}

The median age of the patients was 46 years (13). Median PFS was prolonged by 3 months in the talazoparib group as compared with the physicians' choice of therapy (8.6 vs. 5.6 months, respectively; $\mathrm{P}<0.0001$ ). Improved clinical benefit of talazoparib was observed in all subsets including those with CNS metastases (13). Talazoparib also improved median PFS across all the subgroups of mBC patients (ITT, TNBC and HR+/HER2-) versus standard therapy, irrespective of the number of prior lines of chemotherapy (0L CT, $1 \mathrm{~L}$ CT, $\geq 2 \mathrm{~L}$ CT; where $\mathrm{L}=$ line 
and CT=chemotherapy); the improvement in PFS was statistically significant in patients who had not received prior chemotherapy (0L CT) (14).

There was an improvement in the secondary endpoints; $\mathrm{CR}$ was achieved in $5.5 \%$ patients in the talazoparib group and none in the standard therapy group. The ORR at 24 weeks was observed in $62.6 \%$ patients in the talazoparib group and $27.2 \%$ patients in the standard therapy group. Median duration of response was 5.4 and 3.1 months in the talazoparib and standard therapy groups, respectively (13). There was also an improvement in interim OS in the talazoparib group over standard therapy (22.3 vs. 19.5 months, respectively) (13).

To summarize, treatment with PARPi such as olaparib and talazoparib in OLYMPIAD and EMBRACA, respectively, was found to be associated with an improvement in PFS and response rates compared to the standard therapy of care in patients with HER2- mBC and $\mathrm{g} B R C A$ mutations, especially in those with no prior chemotherapy. These results are encouraging and suggest the use of PARPi earlier on in these patients' course of treatment. However, it is important to optimize $B R C A$ testing in $\mathrm{mBC}$ settings and define the selection and sequencing of PARPi in patients with HER2 - mBC to further enhance outcomes and guide clinical decisionmaking among these patients.

\section{Optimizing BRCA testing among patients with mBC: addressing key clinical questions}

Clinical Question 1: Should all patients with mBC be tested for $g B R C A$ ? At what time point after $m B C$ diagnosis should patients be tested?

A high proportion of individuals who carry a $B R C A$ mutation include those who have been diagnosed with metastatic TNBC (15), while 10-20\% have estrogen receptor-positive (ER+) tumors (16). A retrospective study on 450 TNBC patients undergoing testing for $B R C A$ mutations showed $B R C A 1 / 2$ mutations in about $30.8 \%$ of cases (17). Additionally, the probability of $\mathrm{g} B R C A$ mutation risk has been predicted to be $>10 \%$ in women diagnosed before 50 years of age; thus, the National Comprehensive Cancer Network (NCCN) guidelines strongly recommend consideration of $\mathrm{g} B R C A 1 / 2$ testing in all breast cancer patients below the age of 45 years; in women diagnosed with TNBC at $\leq 60$ years of age, irrespective of family history; and in those with HER2- tumors who are under consideration for chemotherapy as this may have an early survival advantage in $B R C A$ carriers (18-22). The Fourth European Society for Medical Oncology (ESMO) advanced breast cancer $(\mathrm{ABC} 4)$ guidelines state that "in the $\mathrm{ABC}$ setting, results from $\mathrm{g} B R C A 1 / 2$ genetic testing may have therapeutic implications and should therefore be considered as early as possible during the treatment process in these patients" (23).

After a detailed discussion on this clinical question, the panel recommended that ideally all patients with HER2- mBC should undergo BRCA testing. However, in a resource-constrained environment in order to optimize the utilization of resource, consideration can be made to restrict $B R C A$ testing to $\mathrm{mBC}$ patients aged $\leq 50$ years (and to $\leq 60$ years in TNBC settings). The expert panel also concluded that HER2 - mBC patients, especially younger women, should be tested upfront for $\mathrm{g} B R C A$ mutations because there is a therapeutic option (PARPi) available for these patients $(10,13,14)$. In $\mathrm{HR}+\mathrm{mBC}$ patients, $\mathrm{g} B R C A$ testing may be delayed while the patient is being treated with first-line ET. However, it is recommended to consider $\mathrm{g} B R C A$ testing as early as possible even in these patients so that sequencing strategies can be planned and optimized.

\section{Clinical Question 2: Should genetic counselling be mandatory prior to testing in patients with $m B C$ ?}

The process of genetic assessment and counselling includes detailed analysis and risk assessment for potentially harmful genetic mutations. It should also include patient education, discussion of the benefits and harms of genetic testing, discussion of management options and interpretation of results after testing. Genetic counselling for BRCA1/2 mutation testing should be performed by trained healthcare professionals. The US Preventive Services Task Force (USPSTF) recommends that primary care clinicians should assess women with a personal or family history of breast cancer associated with $B R C A 1 / 2$ gene mutations with an appropriate brief familial risk assessment tool. Those with a positive result on the risk assessment tool should then receive genetic counselling and, if appropriate, genetic testing, while those without such an indication should not be offered routine genetic counselling or testing $(24,25)$.

The expert panel discussed and agreed that genetic counselling prior to testing may not be mandatory if not available in limited-resource settings; the clinician may refer the patient to the genetic counsellor, if available, only if genetic testing yields positive results. Alternatively, in the absence of genetic counsellors, the physician should 
Table 1 Status of $B R C A$ testing in the participating regions

\begin{tabular}{|c|c|c|c|}
\hline Participating region & \multicolumn{3}{|c|}{ Status of $B R C A$ testing } \\
\hline Mexico (26) & $\begin{array}{l}B R C A \text { testing is currently not funded, } \\
\text { but there has been increasing awareness } \\
\text { among policy makers considering the } \\
\text { emerging data on the implications of } \\
\text { BRCA testing }\end{array}$ & $\begin{array}{l}B R C A \text { testing is covered } \\
\text { by some private insurance } \\
\text { companies }\end{array}$ & $\begin{array}{l}B R C A \text { testing is done through } \\
\text { NGS as part of some scientific } \\
\text { research projects }\end{array}$ \\
\hline Egypt (33) & - & $\begin{array}{l}B R C A \text { testing is available in } \\
\text { accredited genome labs for a } \\
\text { low cost when compared to } \\
\text { other countries. Turnaround } \\
\text { time for results is also shorter } \\
\text { (10 days for germline and } \\
2 \text { weeks for somatic mutations) }\end{array}$ & $\begin{array}{l}B R C A \text { testing is done through } \\
\text { NGS as part of some clinical } \\
\text { trials in universities }\end{array}$ \\
\hline Colombia & $\begin{array}{l}B R C A \text { testing is covered by the National } \\
\text { Health Insurance, but turnaround time is } 3 \\
\text { months (34) }\end{array}$ & $\begin{array}{l}\text { Genetic testing is generally not } \\
\text { available in South America due } \\
\text { to the high cost (35) }\end{array}$ & - \\
\hline
\end{tabular}

NGS, next generation sequencing.

undertake a genetic counselling session with the patient to address medical implications on personal risk and treatment choices, as well as for family members and future generations.

\section{Clinical Question 3: What is the status of BRCA testing for breast cancer in the participating regions? What are the challenges in BRCA mutation testing?}

Despite the indications of $B R C A$ testing for the management of $\mathrm{mBC}$ patients, there is limited availability of genetic testing in most participating regions. The status of $B R C A$ testing in private, public and research settings in the participating regions is shown in Table 1 (26-35).
In addition to sharing of information regarding the status of $B R C A$ testing among the participating regions, the expert panel listed some common challenges that need to be addressed for scaling-up BRCA testing across all regions, including high cost, long turnover times in obtaining results, and limited government sponsorship/ guidelines for genetic testing. A survey carried out by the Association of Community Cancer Centers (US) revealed similar barriers to routine $B R C A$ mutation testing: (I) patient-related (fear, refusal and concerns for future treatment insurance) and (II) institutional-related (lack of reimbursement for genetic testing/counseling, limited access to genetic counselors and long turnaround time in obtaining test results) (36). 
Clinical Question 4: What are the factors to be considered for identifying pathogenic mutations in mBC patients with $B R C A$ variants of unknown significance?

The rate of $B R C A 1$ or $B R C A 2$ variants of unknown significance (VUS) varies from $2 \%$ to $4 \%(37,38)$. It has been observed that with increasing rates of genetic testing and use of multigene panel testing, the incidence of identification of VUS in breast cancer-related genes continue to increase to reach 20-40\% (39). Many of these VUS may be reclassified over time (40). VUS-related educational interventions for both counselees and referring physicians remain the current need of the hour.

Further to the discussion on this clinical question, the expert panel recommended that if a patient previously identified with $B R C A$ VUS is later diagnosed with recurrent breast cancer, then the physician should consider (depending on the resources available) confirming whether the VUS previously identified has been reclassified as a pathogenic mutation. This process would help in identifying more patients with pathogenic $B R C A$ mutations. Due to lack of enough evidence, currently patients with $B R C A$ VUS should not be considered for PARPi therapy.

\section{Clinical Question 5: Should somatic BRCA testing be contemplated when considering treatment with a PARPi?}

The percentage of somatic $B R C A 1 / 2$ mutations in breast cancer is not well established; however, two studies found that $\sim 3 \%$ of unselected primary breast cancers have a somatic mutation in BRCA1 or BRCA2 $(41,42)$. In breast cancer patients, the rationale for somatic $B R C A 1 / 2$ mutation testing is less developed than for germline testing because of limited data on the efficacy of PARPi for $\mathrm{mBC}$ with somatic $B R C A 1 / 2$ mutations. The expert panel recommended that patients with positive somatic $B R C A$ and negative $g B R C A$ mutations should not be considered for PARPi therapy but may be enrolled into PARPi clinical trials.

\section{Optimizing treatment selection and sequencing of HER2- $\mathrm{mBC}$ in the era of PARP inhibitors: key clinical questions and answers}

Clinical Question 6: Do PARPi trump platinum-based chemotherapy in HER2- breast cancer patients in the metastatic setting?

Currently there are no direct comparisons assessing the efficacy of PARPi versus platinum-based therapy. Olaparib monotherapy in the OLYMPIAD study (10) and talazoparib in the EMBRACA study (13) had a median PFS of 7 and 8.6 months, respectively, in HER2- mBC patients with $\mathrm{g} B R C A$ mutations. The median time from randomization to a second progression event or death following the first progression event was 13.2 months in the olaparib group and 9.3 months in the standard-therapy group in the OLYMPIAD study (HR, 0.57; 95\% CI, 0.40-0.83; $\mathrm{P}=0.003)(10)$. While OLYMPIAD was not powered to demonstrate a statistically significant difference, at the final OS data cut-off ( $64 \%$ maturity), nearly $13 \%$ of patients remained on olaparib and no patients remained on chemotherapy (12). In the phase III TNT trial, patients with $\mathrm{g} B R C A \mathrm{~m}$ HER2-mBC who received first-line carboplatin had a reported PFS of 6.8 months (43). From the reviewed literature, there is indirect evidence that PARPi may have similar efficacy to the much cheaper platinum-based therapy in HER2-, gBRCA mutation-positive patients in the metastatic setting $(9,44)$. The NCCN guidelines also recommend consideration of PARPi monotherapy as an option for HER2-, gBRCA mutation-positive patients (18).

The expert panel, therefore, recommended that if PARPi are unavailable, a platinum-based chemotherapy regimen would be a reasonable option. However, if cost is not an issue, these patients may be initiated with PARPi.

Clinical Question 7: What is the optimal sequencing with PARPi in patients with metastatic TNBC and gBRCA mutation-positive status?

A therapeutic option that is rapidly becoming incorporated in the management of patients with metastatic programmed death-ligand 1 (PDL1)-positive TNBC is the use of immunotherapy drugs such as the combination of atezolizumab plus nab-paclitaxel. The efficacy and safety of this combination was studied in the IMpassion 130 phase III clinical trial.

\section{IMpassion 130: design}

Metastatic TNBC patients (with no prior therapy for metastatic TNBC or with more than 12 months since neoadjuvant/adjuvant therapy) were randomly assigned to receive atezolizumab $(840 \mathrm{mg}$ administered intravenously, on days 1 and 15$)$ plus nab-paclitaxel $\left(100 \mathrm{mg} / \mathrm{m}^{2}\right.$, administered intravenously, on days 1, 8 and 15 of every 28 -day cycle) or nab-paclitaxel alone ( $\mathrm{n}=451$ in each group). Primary endpoints, PFS and OS, were assessed in the ITT and PDL1-positive subgroups (45). 


\section{IMpassion 130: results}

The combination of atezolizumab plus nab-paclitaxel led to significantly longer median PFS in both the ITT population (7.2 vs. 5.5 months; $\mathrm{P}=0.0025)$ and patients with PDLI-positive tumors (7.5 vs. 5.0 months; $\mathrm{P}<0.001$ ) in comparison with nab-paclitaxel alone. In the ITT analysis, median OS was 21.3 months with atezolizumab plus nab-paclitaxel and 17.6 months with nab-paclitaxel alone (HR $=0.84$ ); among patients with PDL1-positive tumors, median OS was 25.0 and 15.5 months, respectively $(\mathrm{HR}=0.62)(45)$. The median duration of response in the ITT population was 7.4 months with atezolizumabnab-paclitaxel and 5.6 months with nab-paclitaxel, while in the PDL1-positive subgroup, it was 8.5 months with atezolizumab-nab-paclitaxel and 5.5 months with placebonab-paclitaxel (45). Exploratory data of the IMpassion 130 study revealed that the clinical benefit derived for PDL1positive metastatic TNBC patients was independent of their $B R C A 1 / 2$ mutation status (46).

Another potential therapeutic target for TNBC is the androgen receptor (AR) (47). However, as per the ESMO guidelines [2018], until the determination of the AR is optimized and standardized, anti-androgen therapy should not be used in routine clinical practice (23). In advanced TNBC settings, the therapeutic efficacy of phosphoinositide 3-kinase (PI3K)/protein kinase B (AKT)/mechanistic target of rapamycin (mTOR) (48), vascular endothelial growth factor (VEGF) (49), tumor protein 53 (p53) (50), checkpoint kinase 1 (CHK1) (51) and WNT signalling (52) targets have also been studied, but these mono-targeted therapies have not yet shown any significant benefit in this setting, due to intrinsic or acquired resistance (53).

The key recommendations from NCCN guidelines for the treatment of HER2 - mBC patients in metastatic TNBC settings are shown in Box 1 .

Based on the reviewed evidence, the expert panel

Box 1 Recommendations of National Comprehensive Cancer Network (NCCN) guidelines [2019] for metastatic triple negative breast cancer (TNBC) patients

The patient's PDL1 expression status on tumor-infiltrating immune cells should be taken into consideration to inform treatment choices for patients with metastatic TNBC

Atezolizumab plus nab-paclitaxel can be considered as an option in PDL1-positive TNBC patients

PARP inhibitors (such as olaparib and talazoparib) may be considered as an option for patients with HER2- tumors and $g B R C A$ mutations

Platinum therapies (such as carboplatin and cisplatin) may be considered as an option for patients with TNBC and gBRCA1/2 mutations

proposed a treatment sequencing algorithm for the management of metastatic TNBC patients (Figure 1). The panel also recommended considering the following aspects while optimizing treatment selection and sequencing therapies in these patients.

All patients with metastatic TNBC should have $g B R C A$ testing upfront.

Patients with stage IV denovo TNBC or recurrent TNBC and disease-free survival (DFS) $\geq 12$ months should have their tumors tested for PDL1.

* In gBRCA mutation-positive metastatic TNBC patients with PDL1-negative status and no visceral crisis, PARPi can be considered for first line of therapy. If there are financial or accessibility constraints or long turnaround times for the genetic testing results, then chemotherapy should be considered.

* In gBRCA mutation-positive metastatic TNBC patients with PDL1-positive status, atezolizumab and nab paclitaxel can be considered as a first line therapeutic option. PARPi may be considered as a second line option in the absence of visceral crisis.

* If patients with metastatic TNBC and PDL1-negative status who received platinum in the neoadjuvant or adjuvant setting had a DFS $\geq 6$ months, they may be considered for re-challenge with platinum therapy.

\section{Clinical Question 8: What is the optimal sequencing with PARPi in patients with $H R+/ H E R 2-m B C$ and $g B R C A$ mutation-positive status?}

The preferred first-line treatment for HR+/HER2$\mathrm{mBC}$ is ET in most cases, excluding those with visceral crisis or concern or proof for endocrine resistance (23). About $50 \%$ of $\mathrm{HR}+$ patients with $\mathrm{mBC}$ have been reported to develop resistance to ET (54). To overcome the development of endocrine resistance, inhibitors 


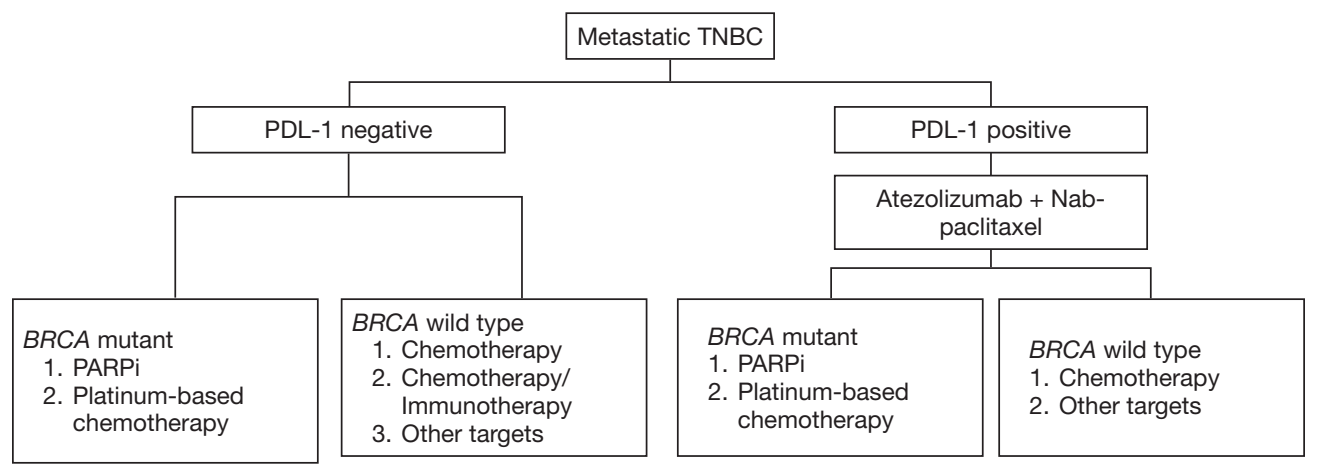

Figure 1 Proposed treatment sequencing for the management of mTNBC. TNBC, triple negative breast cancer; PDL-1, programmed death ligand-1; BRCA, breast cancer gene; PARPi, poly ADP ribose polymerase inhibitor.

of the cyclin-dependent kinases 4 and 6 (CDK4/6) have been evaluated and have recently shown clinically meaningful efficacy in patients with $\mathrm{mBC}$ (55). As evident from landmark clinical trials such as PALOMA-2 (56), MONALEESA-2 (57) and MONARCH-3 (58), the firstline treatment with a CDK4/6 inhibitor (CDK4/6i) plus an aromatase inhibitor (AI) in the endocrine-sensitive $\mathrm{HR}+\mathrm{mBC}$ population led to an approximately 14-month improvement in PFS. Recent data from MONALESSA 3 and 7 have also shown an overall survival advantage with CDK4/6i in the first line setting. In the MONALEESA 3 study, the estimated OS at 42 months was $57.8 \%$ [95\% confidence interval (CI), 52.0-63.2\%] in the ribociclib group and $45.9 \%$ (95\% CI, 36.9-54.5\%) in the placebo group, for a $28 \%$ difference in the relative risk of death in postmenopausal women with HR+/HER2 - mBC (59). In MONALEESA 7, the OS was 70.2\% [95\% confidence interval (CI), 63.5-76.0\%] in the ribociclib group and $46.0 \%$ (95\% CI, $32.0-58.9 \%$ ) in the placebo group in the premenopausal or perimenopausal patients with $\mathrm{HR}+$ / HER2 - mBC (60). Thus, the incorporation of CDK4/6i in combination with ET is an optimal first-line treatment of choice in patients with HR+/HER2- mBC.

Data from the Bolero-2 trial revealed that in endocrineresistant, postmenopausal women with HR+/HER2 $\mathrm{mBC}$ whose disease progressed on prior non-steroidal aromatase inhibitor (AI)-based ET, everolimus (mTOR inhibitor) plus exemestane (AI) doubled the PFS to 7.8 vs. 3.2 months in the exemestane-only group in the second-line setting $(61,62)$. In the global phase III MONARCH-2 trial, fulvestrant (estrogen receptor antagonist) plus CDK4/6 inhibitor therapy in peri/ postmenopausal women with $\mathrm{HR}+/ \mathrm{HER} 2-\mathrm{mBC}$, who progressed on prior ET, increased the median PFS to 16.4 months as compared to 9.3 months with placebo plus fulvestrant (63). Treatment with abemaciclib plus fulvestrant resulted in a statistically significant and clinically meaningful median OS improvement of 9.4 months in patients with advanced HR+/HER2 - mBC who progressed on prior endocrine therapy (64). In the PALOMA 3 trial, patients who had progressed or relapsed on ET alone showed an improvement in the median OS by 6.9 months with CDK4/6i plus fulvestrant therapy (28.0 months with fulvestrant vs. 34.9 months with fulvestrant + palbociclib) in the second-line setting (65). Further, in the MONALEESA 3 study, second-line treatment of $\mathrm{HR}+/ \mathrm{HER} 2-\mathrm{mBC}$ patients with ribociclib + fulvestrant and placebo + fulvestrant group resulted in a median overall survival of 40.2 and 32.5 months, respectively (95\% CI, 0.53-1.00) (66). Thus, it is evident from the reviewed literature that in an endocrine resistant cohort there is an overall survival advantage in patients with HR+/HER2- mBC with CDK4/6i plus endocrine therapy in the second line setting.

In a study assessing the real-world treatment patterns and efficacy of CDK4/6 inhibitors, patients with $\mathrm{g} B R C A$ mutations and $\mathrm{g} B R C A \mathrm{wt}$ status received letrozole plus palbociclib, fulvestrant plus palbociclib, or other CDK4/6 inhibitor regimens across all lines. Patients with $\mathrm{g} B R C A$ mutations had a non-significant, shorter time to first subsequent therapy or death as compared to the gBRCAwt group (11 vs. 14 months). Overall survival was also significantly shorter in patients with $\mathrm{g} B R C A$ mutations versus $\mathrm{g} B R C A \mathrm{wt}$ patients. This study suggested that treatment outcomes with CDK4/6 inhibitors may be poorer in $\mathrm{mBC}$ patients with $\mathrm{g} B R C A$ mutations compared to those 
Box 2 Recommendations of NCCN guidelines (2019) for HR+/HER2 - mBC patients

In patients with visceral crisis, consider initial chemotherapy and continue therapy until progression.

- In patients with no visceral crisis and prior ET within 1 year: (I) in premenopausal women, consider ovarian ablation or suppression plus a different ET \pm CDK4/6 inhibitor or mTOR inhibitor, and continue therapy until disease progression; (II) in postmenopausal women, consider a different ET \pm CDK4/6 inhibitor or mTOR inhibitor and continue therapy until disease progression.

- In patients with no visceral crisis and no prior ET within 1 year: (I) in premenopausal women, consider ovarian ablation or suppression plus ET \pm CDK4/6 inhibitor or mTOR inhibitor, and continue therapy until disease progression; (II) in postmenopausal women, consider an $\mathrm{Al}+\mathrm{CDK} 4 / 6$ inhibitor or fulvestrant + CDK4/6 inhibitor and continue therapy until disease progression.

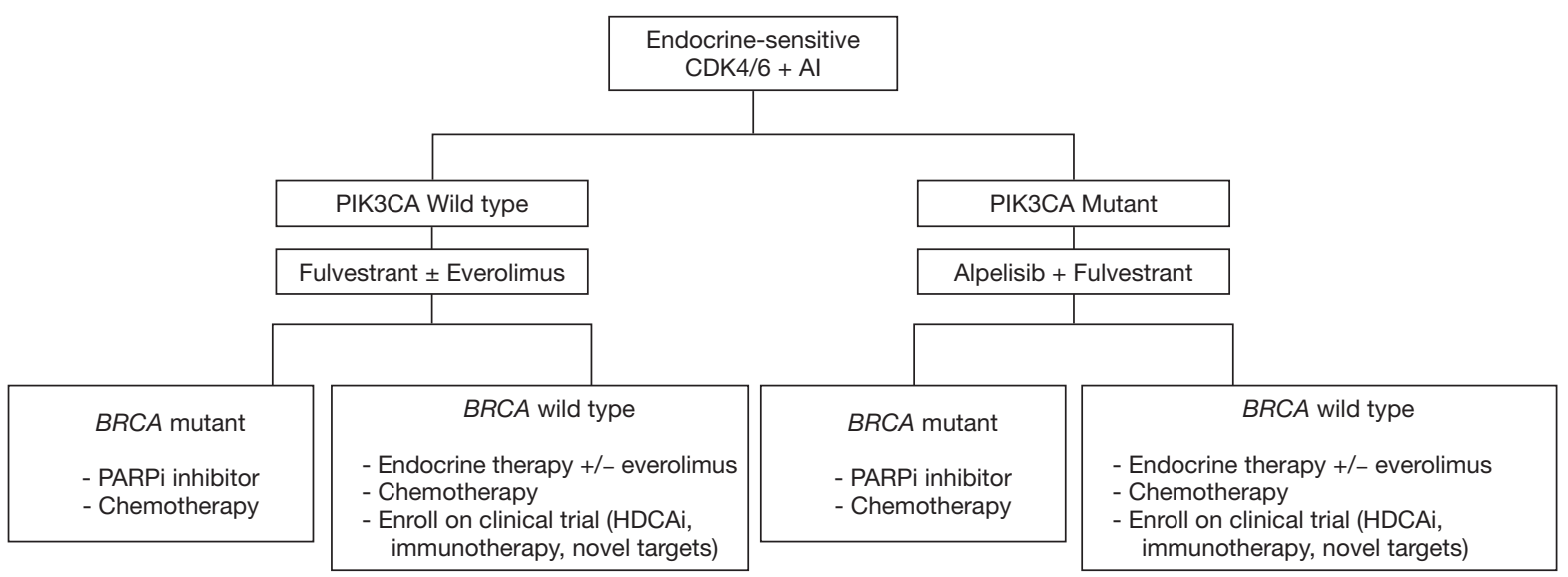

Figure 2 Proposed treatment sequencing algorithm for the management of HR+/HER2 - mBC patients (endocrine-sensitive). CDK4/6i + $\mathrm{AI}$ may be considered as first-line option in these patients, if available and the patients is not in visceral crisis. Selected few patients may not require CDK4/6i in first line and may use it as the second line treatment option. Chemotherapy, eribulin/taxane/capecitabine; PIK3CA, phosphoinositide-3-kinase catalytic alpha polypeptide; AI, aromatase inhibitor; CDK, cyclin-dependent kinase; PARPi, poly ADP ribose polymerase inhibitor.

with $\mathrm{g} B R C A$ wt disease. Further prospective studies are needed to evaluate alternative treatment options in such patient population (67).

Phosphatidylinositol-3-kinase (PI3K) inhibitors are another class of agents explored for the treatment of $\mathrm{HR}+$ /HER2 $-\mathrm{mBC}$ in the second-line setting. In the SOLAR-1 phase III clinical trial, in the cohort of PIK3CAmutated $\mathrm{HR}+$ /HER2 - mBC patients that progressed on prior ET, PFS at a median follow-up of 20 months was 11.0 months in the alpelisib-fulvestrant group, as compared to 5.7 months in the placebo-fulvestrant group (68).

The key recommendations from the NCCN guidelines for the treatment of $\mathrm{HR}+$ /HER2 - mBC patients are shown in Box 2.

Based on the reviewed literature, the expert panel proposed treatment sequencing algorithms for the management of $\mathrm{HR}+\mathrm{mBC}$ patients (endocrine-sensitive, Figure 2; endocrine-resistant, Figure 3).
Clinical Question 9: Is there a need for generating realworld data or a breast cancer registry for HER2- mBC settings?

The expert panel discussed the availability of breast cancer registries in the participating regions. Russia has regional cancer registries across several regions of the Russian Federation which are maintained in the central Cancer Register Information and Analytical System (Cancer Register IAS) (69). As for Argentina, an online hospitalbased registry, known as the Institutional Cancer Registry of Argentina (RITA) is active (70). In Mexico, the Ministry of Health is working towards the establishment of the National Cancer Registry by gathering information on new cancer cases in different regions of the country (71). Population-based cancer registries are currently in development and evolving in Colombia and Egypt (72,73). However there continues to be a gap in the availability 


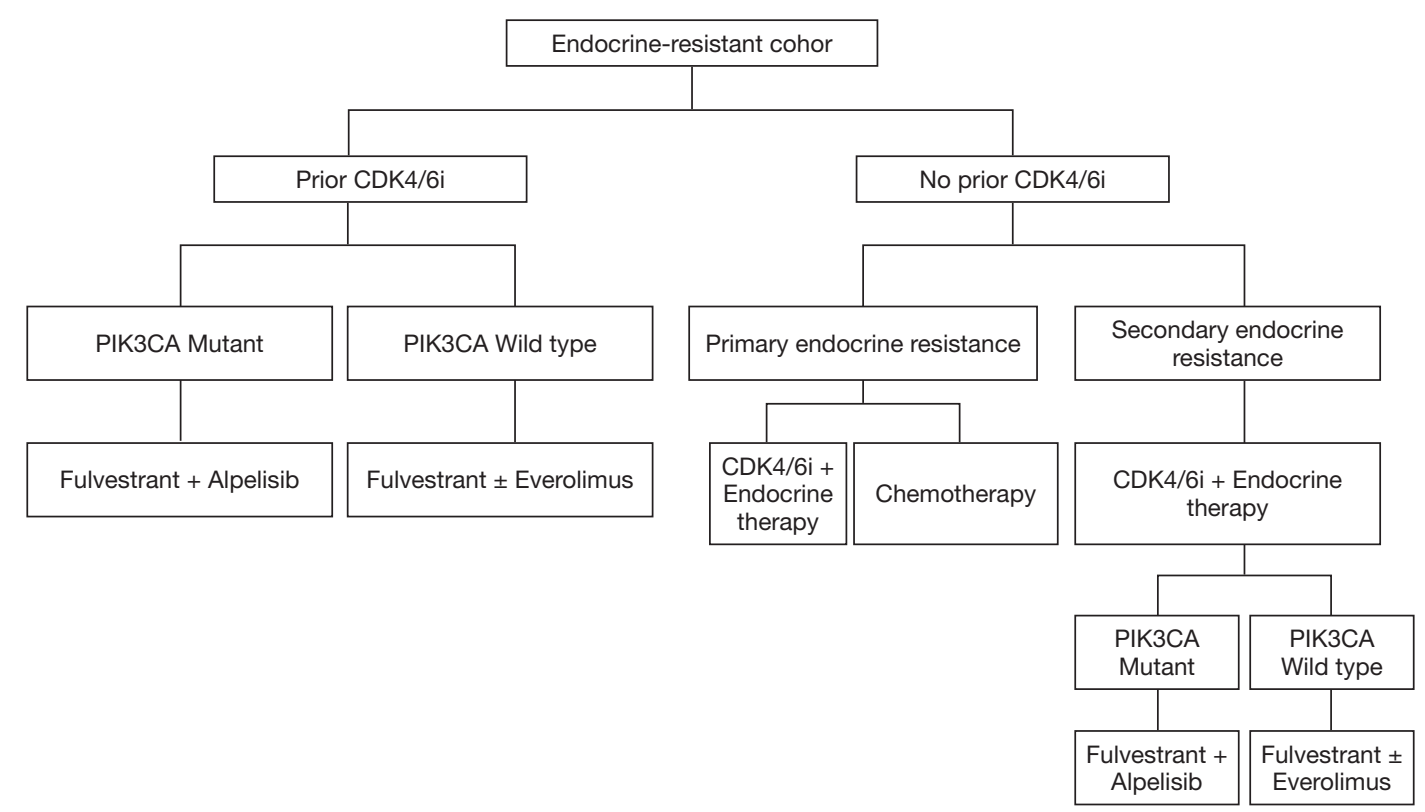

Figure 3 Proposed treatment sequencing algorithm for the management of HR+/HER2- mBC patients (endocrine-resistant). An additional box may be included with BRCAm and PARPi as an option under: (I) prior CDK4/i, PIK3CA wild type; (II) no prior CDK4/6i, primary endocrine resistance, and progressed after CDK4/6i + endocrine therapy (chemotherapy may be used if PARPi is not available); and (III) no prior CDK4/6i, secondary endocrine resistance, and progressed after CDK4/6i + endocrine therapy. Single agent abemaciclib may be used in patients who have progressed after several lines of treatment. PIK3CA, Phosphoinositide-3-kinase catalytic alpha polypeptide; CDK, cyclin-dependent kinase; i, inhibitor.

of real-world data for understanding the clinical practice patterns in these regions.

Based on the regional experience sharing, the expert panel discussed and agreed upon the need for simple, regional, electronic and prospective breast cancer registries for recording patient demographics, tumor biological characteristics, diagnostic practices, types of treatment, line of treatment and details of disease progression. The creation of this registry could provide information to help understand and optimize the clinical practice patterns for the management of HER2- $\mathrm{mBC}$ in the participating regions.

\section{Summary and conclusions}

This article provides evidence-based answers, along with expert views for optimizing the $B R C A$ testing, and treatment selection and sequencing for the management of HER2$\mathrm{mBC}$ in the era of PARPi. While, it may be optimal to offer $g B R C A$ testing to all HER2 - $\mathrm{mBC}$ and TNBC patients aged $\leq 50$ and $\leq 60$ years, respectively, testing for $B R C A$ mutations may be delayed in patients with HR+/HER2-
$\mathrm{mBC}$ by initiating first-line treatment with endocrine therapy. Single-agent PARPi therapy is effective and well tolerated in $g B R C A$-mutated HER2- mBC with similar efficacy to platinum therapy and may be a viable option in these patients, if cost is not a constraint. In $g B R C A$ mutated TNBC patients with PDL1-negative status, while PARPi can be considered as the first choice of treatment, platinum therapy may be considered in patients with prior platinum therapy in neoadjuvant or adjuvant settings and a disease-free interval $\geq 6$ months. Whereas, in patients with PDL1-positive status, atezolizumab and nab paclitaxel is the first-line treatment of choice with PARPi reserved for second-line settings. The role of CDK4/6i in both firstand second-line treatment of HR+HER2- mBC patients, especially those with $w t B R C A$ status has been found to be promising. Although the optimal sequence for treatment of HER2 - mBC is yet to be determined, PARPi therapy can now be considered in either the first or subsequent line settings in patients with $g B R C A$ mutation-positive HER2$\mathrm{mBC}$. The algorithms proposed in this paper may help guide clinicians further optimize the treatment sequence in these patients, while also considering regional factors 
such as availability, accessibility, cost, regulatory approval status, and patients' preference. Furthermore, determining BRCA1/2 mutation status in HER2 - mBC patients may help expand treatment options and should be incorporated as a standard procedure in the new area of cancer care.

\section{Acknowledgments}

We would like to thank AstraZeneca for providing medical writing assistance for the development of this manuscript. We would also like to thank BioQuest Solutions for providing editorial support.

Funding: None.

\section{Footnote}

Provenance and Peer Review: This article was commissioned by the Guest Editor (Shaheenah Dawood) for the series "Targeting the DNA Damaging Pathway: PARPi and Beyond" published in Chinese Clinical Oncology. The article was sent for external peer review organized by the Guest Editor and the editorial office.

Conflicts of Interest: The authors have completed the ICMJE uniform disclosure form (available at http:// dx. doi. org/10.21037/cco-20-138). The series "Targeting the DNA Damaging Pathway: PARPi and Beyond" was commissioned by the editorial office without any funding or sponsorship. SD served as the unpaid Guest Editor of the series, and serves as an unpaid editorial board member of Chinese Clinical Oncology from May 2019 to April 2021. SD reports other from Honoraria from Novartis, BioCon, AstraZeneca, BMS and MSD, Roche and Newbridge, outside the submitted work. MK reports grants and other from Astra Zeneca, Ciba-Geigy AG, GSK, Orion Pharma, Roche, Novartis, and Servier for clinical research, is a speaker for Astra Zeneca, Roche, Novartis, Orion Pfizer, Sanofi, Bristol-Myers Squibb, Servier, Orion Pharma, and Eisai and is a member of the Advisory board for Astra Zeneca, Roche, Novartis, Pfizer, outside the submitted work. FP reports other from Bayer, Pfizer, Astra Zeneca, Roche, Merck Serono, Janssen, and Novartis; and is a speaker for Astra Zeneca, Bayer, Janssen, Merck Serono, and Novartis., outside the submitted work. SBK reports other from Novartis, Sanofi-Aventis, Kyowa-Kirin, and Dongkook Pharma and has a consultant/advisory role at Novartis, Lilly, Enzychem, and ISU ABXIS Co Ltd., outside the submitted work. CVG reports other from
Roche Mexico, AstraZeneca, Novartis, and Myriad, outside the submitted work. SXF reports other from Pfizer, Roche, Novartis, Merck, and Abbvie; is an advisory board member of Pfizer, Roche, Novartis, and Astra-Zeneca; and is a speaker at events for Roche, Novartis, Pfizer, and AstraZeneca, outside the submitted work. The other authors have no other conflicts of interest to declare.

Ethical Statement: The authors are accountable for all aspects of the work in ensuring that questions related to the accuracy or integrity of any part of the work are appropriately investigated and resolved.

Open Access Statement: This is an Open Access article distributed in accordance with the Creative Commons Attribution-NonCommercial-NoDerivs 4.0 International License (CC BY-NC-ND 4.0), which permits the noncommercial replication and distribution of the article with the strict proviso that no changes or edits are made and the original work is properly cited (including links to both the formal publication through the relevant DOI and the license). See: https://creativecommons.org/licenses/by-nc-nd/4.0/.

\section{References}

1. Bray F, Ferlay J, Soerjomataram I, et al. Global cancer statistics 2018: GLOBOCAN estimates of incidence and mortality worldwide for 36 Cancers in 185 countries. CA Cancer J Clin 2018;68:394-24.

2. Cancer Stats Facts: Female Breast Cancer. Available online: https://seer.cancer.gov/statfacts/html/breast.html

3. O'Shaughnessy J. Extending survival with chemotherapy in Metastatic Breast Cancer. Oncologist 2005;10:20-9.

4. Yersal O, Barutca S. Biological subtypes of breast cancer: Prognostic and therapeutic implications. World J Clin Oncol 2014;5:412-24.

5. Cancer Stat Facts: Female Breast Cancer Subtypes. Available online: https://seer.cancer.gov/statfacts/html/ breast-subtypes.html

6. Tutt A, Robson M, Garber JE, et al. Oral poly (ADPribose) polymerase inhibitor olaparib in patients with BRCA1 or BRCA2 mutations and advanced breast cancer: a proof of concept trial. Lancet 2010;376:235-44.

7. Shao N, Shi Y, Yu L, et al. Prospect for Application of PARP Inhibitor in Patients with HER2 Negative Breast Cancer. Int J Biol Sci 2019;15:962-72.

8. Kaufman B, Shapira-Frommer R, Schmutzler RK, et al. Olaparib monotherapy in patients with advanced 
cancer and a germline BRCA1/2 mutation. J Clin Oncol 2015;33:244-50.

9. Turner NC, Telli ML, Rugo HS, et al. A phase II study of talazoparib after platinum or cytotoxic nonplatinum regimens in patients with advanced breast cancer and germline BRCA1/2 mutations (ABRAZO). Clin Cancer Res 2019;25:2717-24.

10. Robson M, Im SA, Senkus E, et al. Olaparib for Metastatic Breast Cancer in Patients with a Germline BRCA Mutation. N Engl J Med 2017;377:523-33.

11. Tung NM, Im SA, Senkus-Konefka E, et al. Olaparib versus chemotherapy treatment of physicians' choice in patients with a germline BRCA mutation and HER2negative metastatic breast cancer (OlympiAD): Efficacy in patients with visceral metastases. J Clin Oncol 2018;36:1052.

12. Robson ME, Tung N, Conte $\mathrm{P}$, et al. OlympiAD final overall survival: olaparib versus chemotherapy treatment of physicians' choice (TPC) in patients with metastatic breast cancer $(\mathrm{mBC})$ and a germline BRCA mutation (gBRCAm). Cancer Res 2018;78:Abstract nr CT038.

13. Litton J, Rugo HS, Ettl J, et al. EMBRACA: A phase 3 trial comparing talazoparib, an oral PARP inhibitor, to physicians' choice of therapy in patients with advanced breast cancer and a germline BRCA-mutation. Cancer Res 2018;78:Abstract nr GS6-07.

14. Ettl J, Hurvitz SA, Rugo HS, et al. Outcomes of talazoparib (TALA) versus physician's choice of chemotherapy (PCT) in patients (pts) with advanced breast cancer $(\mathrm{ABC})$ and a germline BRCA (gBRCA) mutation by line of chemotherapy (CT) in the EMBRACA trial. J Clin Oncol 2019;37:1071.

15. Armstrong N, Ryder S, Forbes C, et al. A systematic review of the international prevalence of BRCA mutation in breast cancer. Clin Epidemiol 2019;11:543-61.

16. Kaplan JS, Schnitt SJ, Collins LC, et al. Pathologic features and immunophenotype of estrogen receptorpositive breast cancers in BRCA1 mutation carriers. Am J Surg Pathol 2012;36:1483-8.

17. Greenup R, Buchanan A, Lorizio W, et al. Prevalence of BRCA mutations among women with triple negative breast cancer (TNBC) in a genetic counseling cohort. Ann Surg Oncol 2013;20:3254-8.

18. NCCN clinical practice guidelines in oncology. Breast cancer. Version 3.2019. Available online: https://www. nccn.org/professionals/physician_gls/\#site

19. Engel C, Rhiem K, Hahnen E, et al. Prevalence of pathogenic BRCA1/2 germline mutations among 802 women with unilateral triple negative breast cancer without family cancer history. BMC Cancer 2018;18:265.

20. Rosenberg SM, Ruddy KJ, Tamimi RM, et al. BRCA1 and BRCA2 mutation testing in young women with breast cancer. JAMA Oncol 2016;2:730.

21. Copson ER, Maishman TC, Tapper WJ, et al. Germline BRCA mutation and outcome in young-onset breast cancer (POSH): a prospective cohort study. Lancet Oncol 2018;19:169-80.

22. Tung NM, Garber JE. BRCA1/2 testing: therapeutic implications for breast cancer management. Br J Cancer 2018;119:141-52.

23. Cardoso F, Senkus E, Costa A, et al. 4th ESO-ESMO International Consensus Guidelines for Advanced Breast Cancer (ABC 4). Ann Oncol 2018;29:1634-57.

24. Owens DK, Davidson KW, Krist AH, et al. Risk assessment, genetic counseling, and genetic testing for BRCA-related cancer US preventive services task force recommendation statement. JAMA 2019;322:652-65.

25. Meiser B, Tucker, K, Friedlander M, et al. Genetic counselling and testing for inherited gene mutations in newly diagnosed patients with breast cancer: a review of the existing literature and a proposed research agenda. Breast Cancer Res 2008;10:216.

26. Millan Catalan O, Campos-Parra AD, Vázquez-Romo $\mathrm{R}$, et al. A multi-center study of BRCA1 and BRCA2 germline mutations in Mexican-Mestizo breast cancer families reveals mutations unreported in Latin American population. Cancers (Basel) 2019;11:1246.

27. Fernandes GC, Michelli RAD, Galvão HCR, et al. Prevalence of BRCA1/BRCA2 mutations in a Brazilian population sample at-risk for hereditary breast cancer and characterization of its genetic ancestry. Oncotarget 2016;7:80465-81.

28. Palmero EI, Carraro DM, Alemar B, et al. The germline mutational landscape of BRCA1 and BRCA2 in Brazil. Sci Rep 2018;8:9188.

29. Chavarri-Guerra Y, Blazer KR, Weitzel JN, et al. Genetic cancer risk assessment for breast cancer in Latin America. Rev Invest Clin 2017;69:94-102.

30. Jaure O, Alonso EN, Braico DA, et al. BRCA1 polymorphism in breast cancer patients from Argentina. Oncol Lett 2015;9:845-50.

31. Sun Y, Kang E, Baek H, et al. Participation of Korean families at high risk for hereditary breast and ovarian cancer in BRCA1/2 genetic testing. Jpn J Clin Oncol 2015;45:527-32.

32. Lee EG, Kang HJ, Lim MC, et al. Different patterns 
of risk reducing decisions in affected or unaffected BRCA pathogenic variant carriers. Cancer Res Treat 2019;51:280-8.

33. Ibrahim SS, Hafez EE, Hashishe MM, et al. Presymptomatic breast cancer in Egypt: role of BRCA1 and BRCA2 tumor suppressor genes mutations detection. J Exp Clin Cancer Res 2010;29:82.

34. Briceño-Balcázar I, Gómez-Gutiérrez A, Díaz-Dussán NA, et al. Mutational spectrum in breast cancer associated BRCA1 and BRCA2 genes in Colombia. Colomb Med (Cali) 2017;48:58-63.

35. Hernández JE, Llacuachaqui $M$, Palacio GV, et al. Prevalence of BRCA1 and BRCA2 mutations in unselected breast cancer patients from Medellin, Colombia. Hered Cancer Clin Pract 2014;12:11.

36. Assessing the status of BRCA testing and the challenges faced by cancer care teams in the community. Association of Community Cancer Centers website. Posted May 14, 2018. Available online: https://www.accc-cancer.org/docs/ projects/brca/brca-initiative_survey-summary_final-v2. pdf?sfvrsn=80fe0ffb_2. Accessed June 20, 2019.

37. Grindedal EM, Heramb C, Karsrud I, et al. Current guidelines for BRCA testing of breast cancer patients are insufficient to detect all mutation carriers. BMC Cancer 2017;17:438.

38. Variants of unknown significance. Available online: https:// www.breastcancer.org/symptoms/testing/genetic/variants, Accessed June 20, 2019.

39. Carter NJ, Theobald K, Murphy P, et al. Yield of pathogenic/likely pathogenic variants in breast cancer patients undergoing an inherited cancer panel based upon ethnic background. ACMG 2016 Poster. Available online: https://www.genedx.com/wp-content/uploads/2016/04/ ACMG-2016-Ethnicity-and-IC-Panel-Testing-Results.pdf

40. Welsh JL, Hoskin TL, Day CN, et al. Clinical decisionmaking in patients with variant of uncertain significance in BRCA1 or BRCA2 genes. Ann Surg Oncol 2017;24:3067-72.

41. Nik-Zainal S, Davies H, Staaf J, et al. Landscape of somatic mutations in 560 breast cancer whole-genome sequences. Nature 2016;534:47-54.

42. Winter C, Nilsson MP, Olsson E, et al. Targeted sequencing of BRCA1 and BRCA2 across a large unselected breast cancer cohort suggests that one-third of mutations are somatic. Ann Oncol 2016;27:1532-38.

43. Tutt A, Tovey H, Cheang MCU, et al. Carboplatin in BRCA1/2-mutated and triple-negative breast cancer BRCAness subgroups: The TNT trial. Nat Med
2018;24:628-37.

44. Poggio F, Bruzzone M, Ceppi M, et al. Single agent PARP inhibitors for the treatment of patients with BRCA-mutated HER2-negative metastatic breast cancer: a systematic review and meta-analysis. ESMO Open 2018;3:e:000361.

45. Schmid P, Adams S, Rugo HS, et al. Atezolizumab and Nab-Paclitaxel in Advanced Triple-Negative Breast Cancer. N Engl J Med 2018;379:2108-21.

46. Emens LA, Loi S, Rugo HS, et al. Impassion 130: Efficacy in immune biomarker subgroups from the global, randomized, double-blind, placebo-controlled, phase III study of atezolizumab+nab-paclitaxel in patients with treatment-naïve, locally advanced or metastatic triplenegative breast cancer. Cancer Res 2019;79:Abstract nr GS1-04.

47. Traina TA, Miller K, Yardley DA, et al. Enzalutamide for the treatment of androgen receptor-expressing triplenegative breast cancer. J Clin Oncol 2018;36:884-90.

48. Ganesan P, Moulder S, Lee JJ, et al. Triple-negative breast cancer patients treated at MD Anderson Cancer Center in phase I trials: Improved outcomes with combination chemotherapy and targeted agents. Mol Cancer Ther 2014;13:3175-84.

49. Cameron D, Brown J, Dent R, et al. Adjuvant bevacizumab-containing therapy in triple-negative breast cancer (BEATRICE): primary results of a randomised, Phase III trial. Lancet Oncol 2013;14:933-42.

50. Synnott NC, Murray A, Mcgowan PM, et al. Mutant p53: a novel target for the treatment of patients with triplenegative breast cancer? Int J Cancer 2017;140:234-46.

51. Bryant C, Rawlinson R, Massey AJ, et al. Chk1 Inhibition as a novel therapeutic strategy for treating triple-negative breast and ovarian cancers. BMC Cancer 2014;14:570.

52. Liu J, Pan S, Hsieh MH, et al. Targeting Wnt-driven cancer through the inhibition of porcupine by LGK974. Proc Natl Acad Sci U S A 2013;110:20224-9.

53. Jhan JR, Andrechek ER. Triple negative breast cancer and the potential for targeted therapy. Pharmacogenomics 2017;18:1595-609.

54. Osborne CK, Schiff R. Mechanisms of endocrine resistance in breast cancer. Annu Rev Med 2011;62:233-47.

55. Rossi L, McCartney A, Risi E, et al. Managing Advanced HR-Positive, HER2-Negative Breast Cancer with CDK4/6 Inhibitors in Post-Menopausal Patients: Is There a Best Sequence? Ther Adv Med Oncol 2018;10:1758835918815591. 
56. Rugo HS, Finn RS, Diéras V, et al. Palbociclib plus letrozole as first-line therapy in estrogen receptor-positive/ human epidermal growth factor receptor 2-negative advanced breast cancer with extended follow-up. Breast Cancer Res Treat 2019;174:719-29.

57. Hortobagyi GN, Stemmer SM, Burris HA, et al. Ribociclib as First-Line Therapy for HR-Positive, Advanced Breast Cancer. N Engl J Med 2016;375:1738-48.

58. Goetz MP, Toi M, Campone M, et al. MONARCH 3: Abemaciclib As Initial Therapy for Advanced Breast Cancer. J Clin Oncol 2017;35:3638-46.

59. Slamon DJ, Neven P, Chia S, et al. Overall Survival with Ribociclib plus Fulvestrant in Advanced Breast Cancer. N Engl J Med 2020;382:514-24.

60. Im SA, Lu YS, Bardia A, et al. Overall survival with ribociclib plus endocrine therapy in breast cancer. N Engl J Med 2019;381:307-16.

61. Piccart M, Baselga J, Noguchi S, et al. Final progressionfree survival analysis of BOLERO-2: A phase III trial of Everolimus for postmenopausal women with advanced breast cancer. Cancer Res 2012;72:Abstract nr P6-04-02.

62. Baselga J, Campone M, Piccart M, et al. Everolimus in postmenopausal hormone-receptor-positive advanced breast cancer. N Engl J Med 2012;366:520-9.

63. Sledge GW Jr, Toi M, Neven P, et al. MONARCH 2: Abemaciclib in Combination With Fulvestrant in Women With HR+/HER2- Advanced Breast Cancer Who Had Progressed While Receiving Endocrine Therapy. J Clin Oncol 2017;35:2875-84.

64. Sledge GW, Toi M, Neven P, et al. The Effect of Abemaciclib Plus Fulvestrant on Overall Survival in Hormone Receptor-Positive, ERBB2-Negative Breast Cancer That Progressed on Endocrine TherapyMONARCH 2: A Randomized Clinical Trial. JAMA

Cite this article as: Dawood S, Konstantinova M, Perazzo F, Kim SB, Villarreal-Garza C, Franco SX, Simon SD, El-Nahas T. Optimizing the management of HER2-negative metastatic breast cancer in the era of PARP inhibitors-proceedings from breast cancer expert group meeting. Chin Clin Oncol 2020;9(5):61. doi: 10.21037/cco-20-138
Oncol 2019;6:116-24.

65. Turner NC, Slamon DJ, Ro J, et al. Overall survival with Palbociclib and Fulvestrant in advanced breast Cancer. N Engl J Med 2018;379:1926-36.

66. Slamon DJ, Neven P, Chia S, et al. Overall survival (OS) results of the Phase III MONALEESA-3 trial of postmenopausal patients (pts) with hormone receptorpositive $(\mathrm{HR}+)$, human epidermal growth factor 2-negative (HER2-) advanced breast cancer (ABC) treated with fulvestrant $(\mathrm{FUL}) \pm$ ribociclib $(\mathrm{RIB})$. Ann Oncol 2019;30:v856-v857.

67. McLaurin K, Dalvi T, Collins JM, et al. A real-world evidence study of CDK4/6 inhibitor treatment patterns and outcomes in metastatic breast cancer by gBRCA mutation status. J Clin Oncol 2019;37:1563.

68. André F, Ciruelos E, Rubovszky G, et al. Alpelisib for PIK3CA-mutated, hormone receptor-positive advanced breast cancer. N Engl J Med 2019;380:1929-40.

69. Andrei K, Starinsky V, Gretsova O, et al. Populationbased registry of cancer patients in the Russian Federation. Public Health Panorama 2019;5:1-121.

70. Abriata MG, Macias GR. Patients with breast cancer: Report from a National hospital-based cancer registry in Argentina, 2012 to 2016. J Glob Oncol 2019;5:1-10.

71. National Cancer Registry. Available online: https://www. gob.mx/salud/prensa/260-iniciara-registro-nacional-decancer. Accessed June 2019.

72. Bravo LE, Munoz N. Epidemiology of cancer in Colombia. Colomb Med (Cali) 2018;49:9-12.

73. Cancer profile in Aswan, Egypt. Available online: http://www.mcit.gov.eg/Upcont/Documents/ Cancer\%20Profile\%20Layout2010526135657.pdf. Accessed June 2019. 